\title{
Semiquantitative evaluation of ophthalmic collateral flow in carotid artery occlusion: ultrasonic doppler
} study

\author{
H IR A O KANEDA, TADAYOSHI IRINO, MANABU WATANABE, \\ EIJ I K A DOTA, A N D M AMORU TANEDA
}

From the Division of Cerebrovascular Diseases, Hanwa Hospital, Osaka, Japan

SUMMARY The reliability of quantitative evaluation by doppler ultrasound with regard to the ophthalmic collateral blood flow in patients with carotid artery occlusion was estimated. The ultrasonic doppler flow signals of the ophthalmic collateral flow of 54 carotid occlusions were classified into four types and three degrees-high, moderate, and low reversed flow patternsand were compared with the angiographic findings of the collateral flow classified into three grades-good, poor, and none. With the exception of three cases, the ultrasonic doppler flow patterns of the collateral flow correlated well with the angiographic findings in 54 occluded carotid arteries.

The clinical symptoms of internal carotid occlusion are various, from no symptom to severe neurological deficit. One of the reasons for the symptomatic variation is the amount of collateral blood flow which flows into the distal territory of the occluded portion of the carotid artery through the ophthalmic artery, the circle of Willis, and/or other arteries. Therefore, it is clinically important to evaluate the collateral flow in patients with carotid artery occlusion. Although angiography is the best method for evaluating the collateral flow, its use is restricted because of its invasive nature and side effects. Much attention has been focused on the noninvasive evaluation of the collateral flow with the ultrasonic doppler technique.

A patient with carotid occlusion frequently has a collateral flow through the ophthalmic artery which is often detectable as a "reversed" blood flow by the ultrasonic doppler flowmeter. Based on this pathological phenomenon, many authors (Müller, 1973; Hyman, 1974; Katz et al., 1976; Lye et al., 1976; von Reutern et al., 1976) reported that an occlusion or high grade stenosis of the carotid artery had been diagnosed noninvasively using doppler ultrasound. However, quantitative evaluation of the ophthalmic collateral flow by doppler ultrasound has not been

Address for reprint requests: Dr Hirao Kaneda, Hanwa Memorial Hospital, 73-7, Karita-cho, Sumiyoshi-ku, Osaka 558, Japan.

Accepted 21 April 1979 studied previously.

The purpose of the present study was to evaluate by comparing the angiograms whether the quantitative findings of the doppler ultrasound are reliable with regard to ophthalmic collateral flow.

\section{Patients and methods}

Fifty-four occluded internal or common carotid arteries of 51 patients ( 20 women, 31 men) were studied. Their mean age was 62.9 years. The clinical presentation ranged from acute to chronic stage within one year of the onset of the stroke.

A directional ultrasonic doppler flowmeter was connected to a probe transmitting an ultrasound frequency of $5 \mathrm{MHz}$. The flowmeter had two filters to separate the signal into two signals corresponding to flow toward probe and flow away from probe. Each blood flow signal was recorded on a magnetic tape recorder and analysed by a sound spectrograph (Rion, SG-07, Tokyo) to produce a sonogram in which we called the maximum blood flow velocity at systole as " $S$ " and that at diastole as " $d$ " (Fig. 1). In the sonogram of reversed ophthalmic blood flow caused by carotid occlusion, we defined the maximum systolic blood flow (S) as a reversed ophthalmic blood flow index for simplification, and we measured this in each patient. 


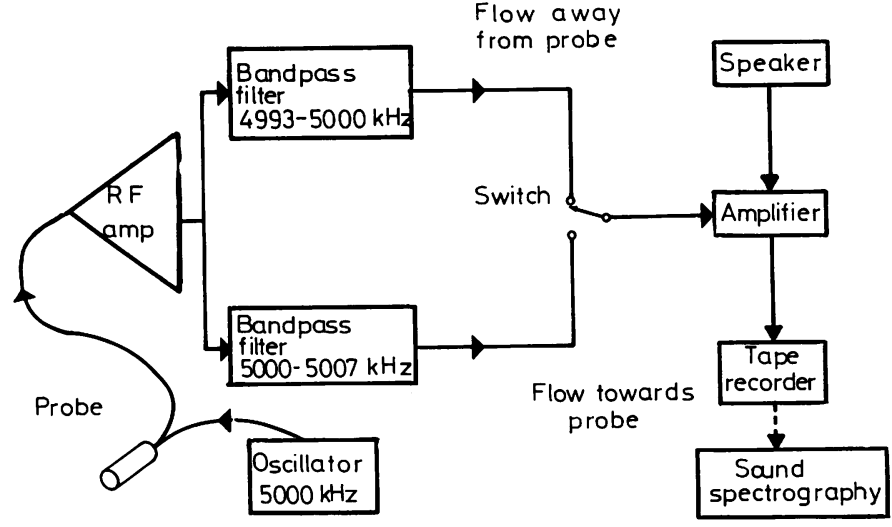

Sonogram of blood flow

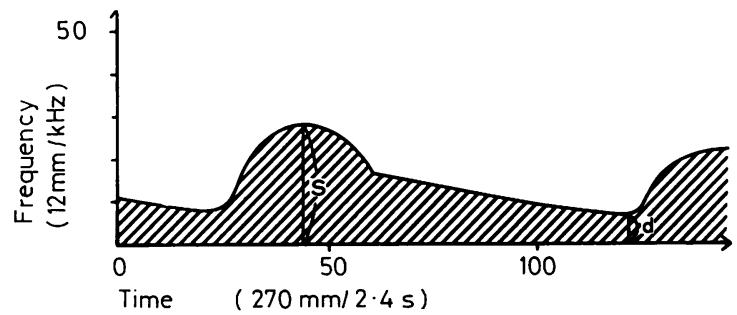

Fig. 1 Block diagram of a directional ultrason:c doppler flowmeter with dual filter type (upper). Illustrative presentation of a sonogram of blood flow analysed by the sound spectrograph (lower).
Blood flow in the territory of the ophthalmic artery is detectable in two regions. One is on the skin of the upper eyelid about $10 \mathrm{~mm}$ above and lateral to the corneal midpoint where blood flow in the ophthalmic artery can be detected. The other is on the inner canthus near the orbital fissure where blood flow in the medial frontal artery, a branch of the ophthalmic artery, can be detected. The former technique was adopted in the present study because it is more informative than the medial frontal artery blood flow detection (Kaneda et al., 1978).

Carotid angiograms were made in all subjects, using $7.5 \mathrm{ml}$ of $60 \%$ amidotrizoate as contrast medium, injected into the common carotid artery at a speed of $7.5 \mathrm{ml} /$ second. Serial angiograms were obtained using an automatic injector and an automatic cassette changer. Intracranial filling of the contrast medium through the ophthalmic artery was carefully observed to determine the quantity of this collateral blood flow.

If more than one angiogram or doppler examination was performed in a patient, one angiogram and one doppler study with the shortest time interval were chosen and compared.

\section{Results}

\section{CAROTID ANGIOGRAPHY}

Ophthalmic collateral flow associated with carotid artery occlusion was classified into three grades. When the contrast medium flowed into the middle cerebral artery and its branches through the ophthalmic artery as shown in Fig. 2, the flow was defined as "good collateral flow." If the contrast medium only filled to the syphon of the internal carotid artery or to the stem of the middle cerebral artery without filling its branches (Fig. 3), the flow was defined "poor ophthalmic collateral flow." When there was no collateral flow via the ophthalmic artery (Fig. 4), the definition was "no ophthalmic collateral flow."

There were seven patients with good ophthalmic collateral flow, 10 with poor collateral flow, and 37 with no collateral flow.

\section{DOPPLER ULTRASOUND}

Sonograms of the reversed ophthalmic blood flow of the 32 patients were arranged according to the value of " $S$ " from high degree to low (Fig. 5). The results were then divided into three degrees. 


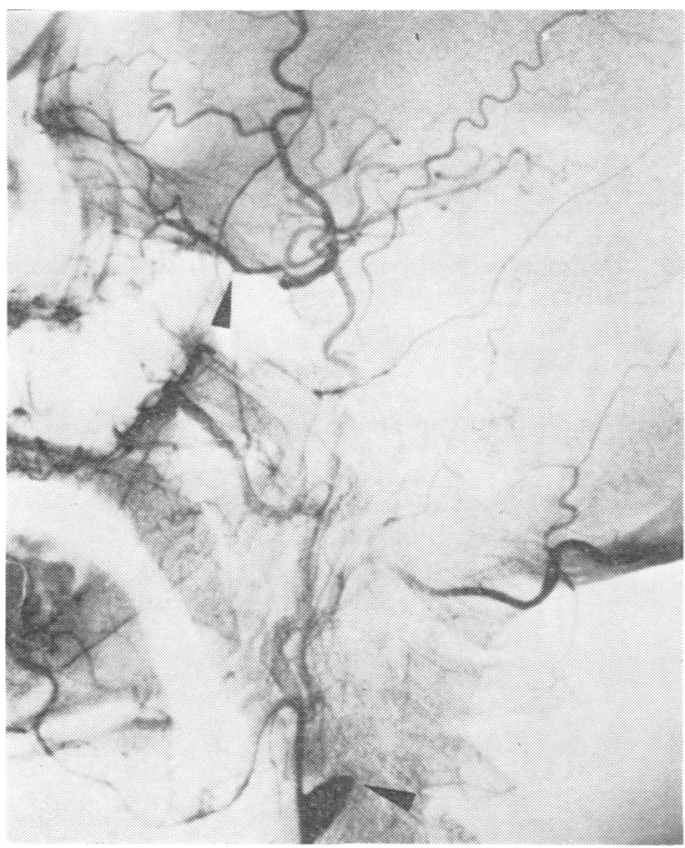

Fig. 2 Angiogram of a 65 year old man showing an internal carotid occlusion (lower arrow) and a good collateral flow through the ophthalmic artery (upper arrow).

That higher than $38 \mathrm{~mm}(=3.17 \mathrm{kHz})$ was defined as a "high reversed flow"; from $19 \mathrm{~mm}$ $(=1.58 \mathrm{kHz})$ to $38 \mathrm{~mm}(=3.17 \mathrm{kHz})$ was classified as a "moderate reversed flow"; and lower than $19 \mathrm{~mm}(=1.58 \mathrm{kHz})$ was termed a "low reversed flow." Typical sonograms of the high, moderate, and low reversed ophthalmic collateral flow are

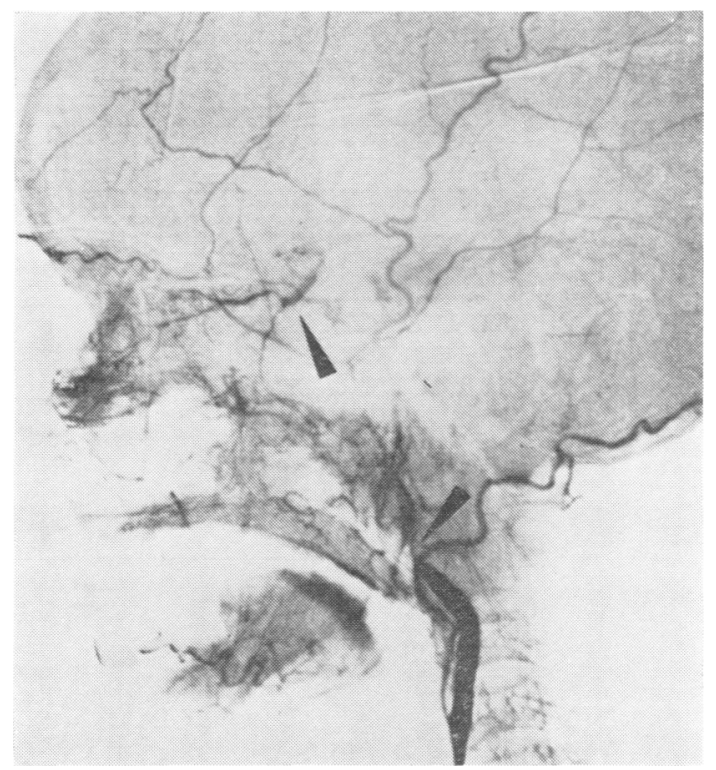

Fig. 3 The ophthalmic collateral flow extended to the syphon of the internal carotid artery and the stem of the middle cerebral artery (upper arrow) but did not fill its branches ( 72 year old woman).

represented in Fig. 6. There were eight patients with high reversed flow, eight with moderate reversed flow, and 16 with low reversed flow. The other 22 patients did not have a reversed ophthalmic flow. Thirteen patients had no flow signal type, two patients had a mixed type of flow, and seven patients had a physiological type of flow according to the ultrasonic doppler study (Fig. 7).

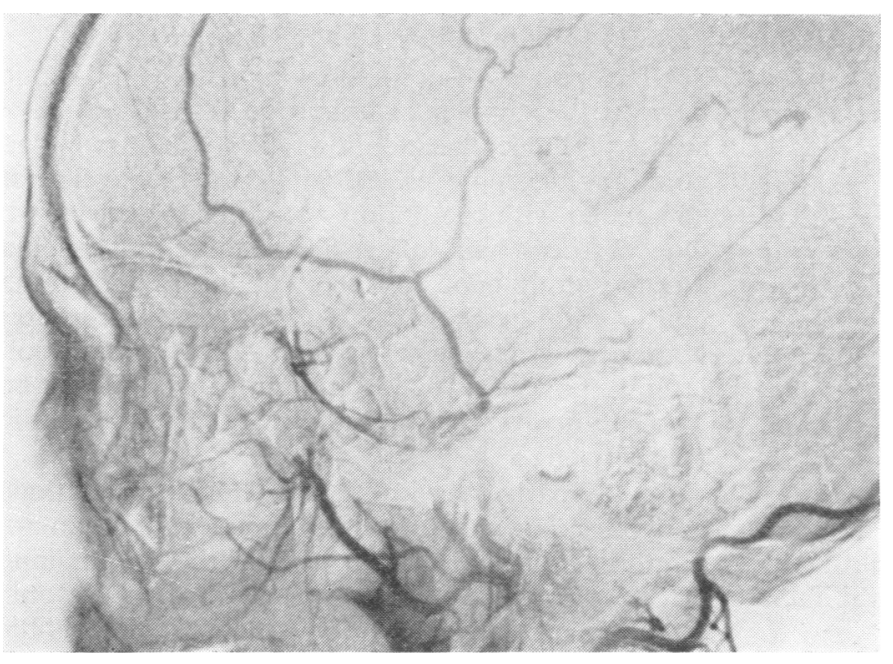

Fig. 4 Angiogram showing collateral flow not through the ophthalmic artery but, to a slight degree, through the middle meningeal artery ( 52 year old man). 


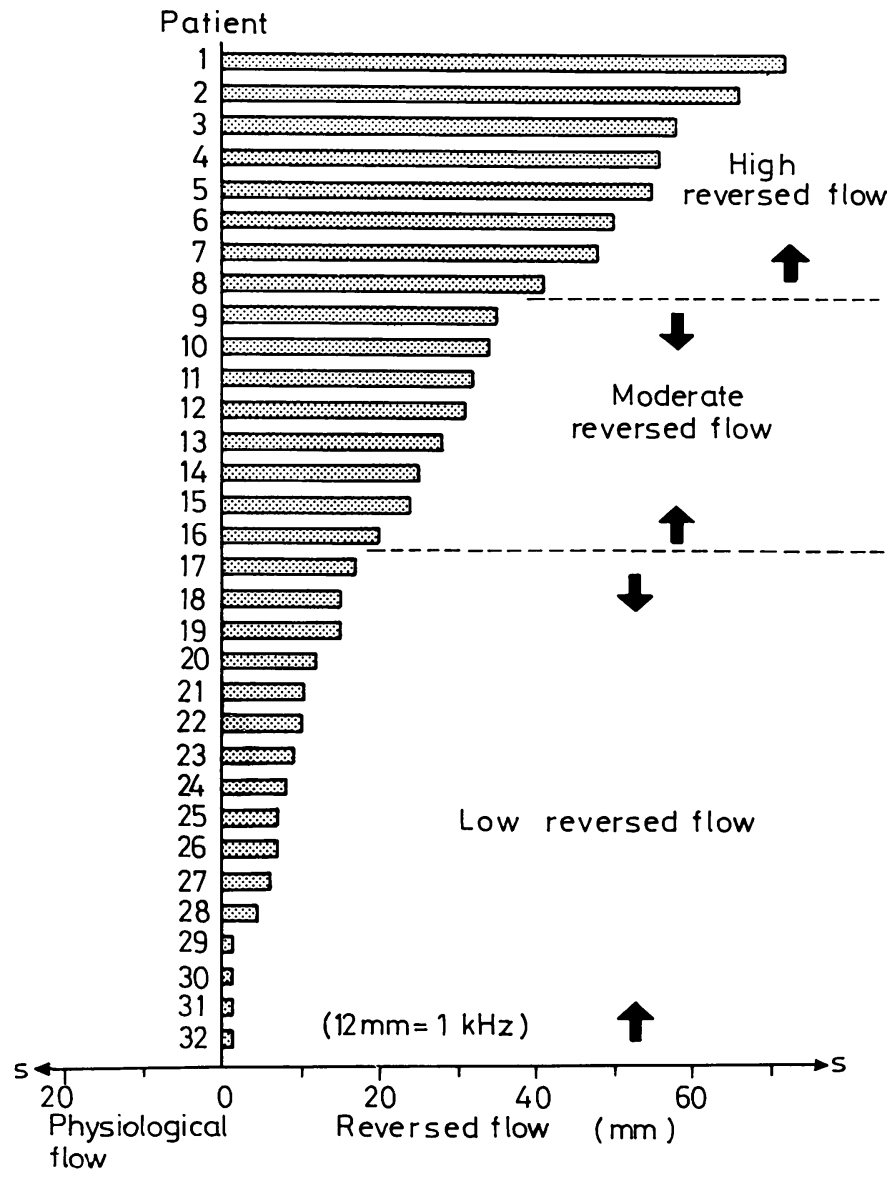

Fig. 5 Quantitative evaluation by doppler ultrasound of reversed ophthalmic flow of the 32 carotid occlusions.

COMPARISON OF ULTRASONIC DOPPLER AND ANGIOGRAPHIC FINDINGS

Ultrasonic doppler findings on ophthalmic collateral flow associated with 54 occluded carotid arteries were compared with the angiographic findings (Fig. 8).

Eight patients with high reversed ophthalmic flow on doppler ultrasound all had a good angiographic collateral flow through the ophthalmic artery, except for one patient whose carotid angiograms did not show any ophthalmic collateral flow. The reason for the absence of collateral flow on angiography in this case was that the high reversed ophthalmic flow was filled across the midline of the face, via the superficial arteries of the face from the contralateral external carotid artery. Digital compression of the contralateral common carotid artery stopped this reversed ophthalmic flow and compression of the contralateral facial artery decreased it.
Eight patients with moderate reversed flow according to the doppler study had a poor angiographic collateral flow without exception.

Of 16 patients who had low reversed ophthalmic flow on the doppler examination, 14 did not have angiographic collateral flow. The other two had poor angiographic collateral flow which was not correlated with the findings of doppler ultrasound. One of them had suffered from cerebral oedema due to simultaneous chronic subdural haematoma and internal carotid occlusion to the same cerebral hemisphere. The other patient had the main collateral flow through small arteries near the ophthalmic artery but not through the ophthalmic artery itself.

Of 22 patients with the no flow, mixed flow, and physiological flow types, none had an angiographic collateral flow through the ophthalmic artery. Therefore, the findings of ultrasonic doppler study correlated well with the angiographic 

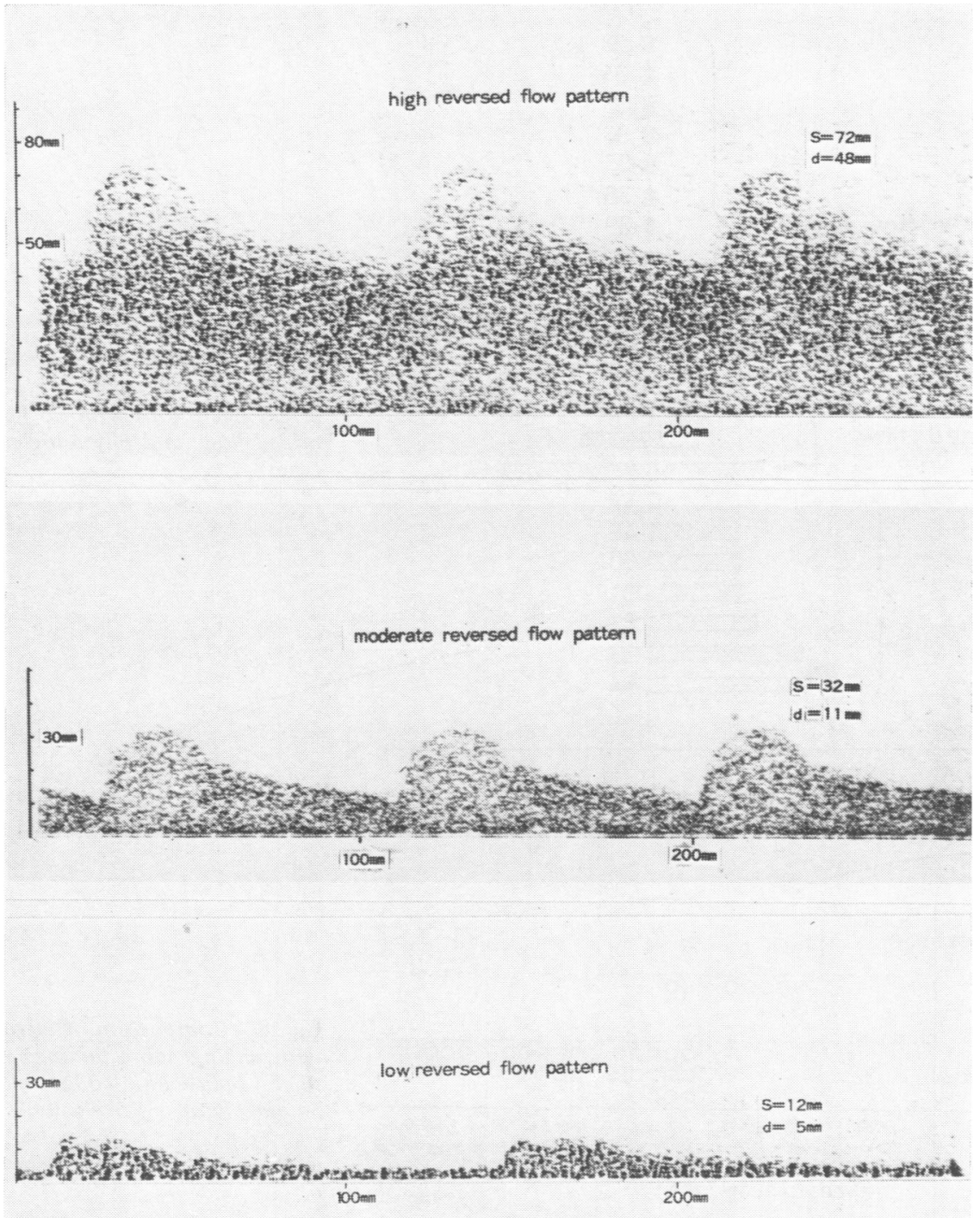

Fig. 6 Typical sonograms of high, moderate, and low reversed ophthalmic blood flow associated with carotid artery occlusion.

findings except for three patients when we evaluated quantitatively the ophthalmic collateral flow in 54 cases of carotid occlusion.

\section{Discussion}

Since the ultrasonic doppler technique was introduced for measurement of blood flow by Satomura and Kaneko in 1960, it has been widely used for the evaluation of blood flow in various arteries. In the field of cerebrovascular diseases, Maroon et al. (1970) and Müller (1972) reported the diagnostic possibility in internal carotid occlusion detecting the direction of blood flow in the territory of the ophthalmic artery. Thereafter many authors have reported its diagnostic usefulness in obstructive lesions of the internal carotid (Keller et al., 1976a; Shoumaker and Bloch, 1978; White and Curry, 1978; Wise et al., 1979) and vertebral artery (Kaneda et al., 1977). 


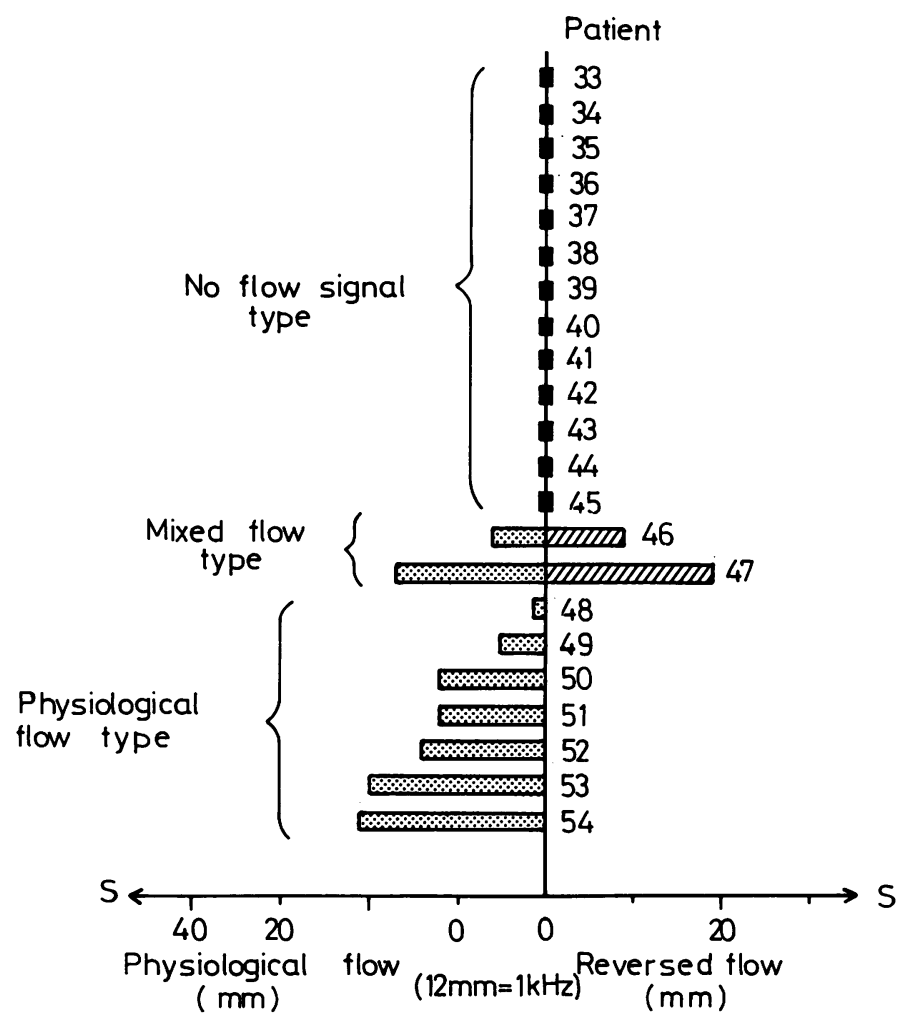

Fig. 7 Ultrasonic doppler findings of the ophthalmic blood flow with 22 carotid artery occlusions with no flow signal, mixed flow, and physiological flow types.

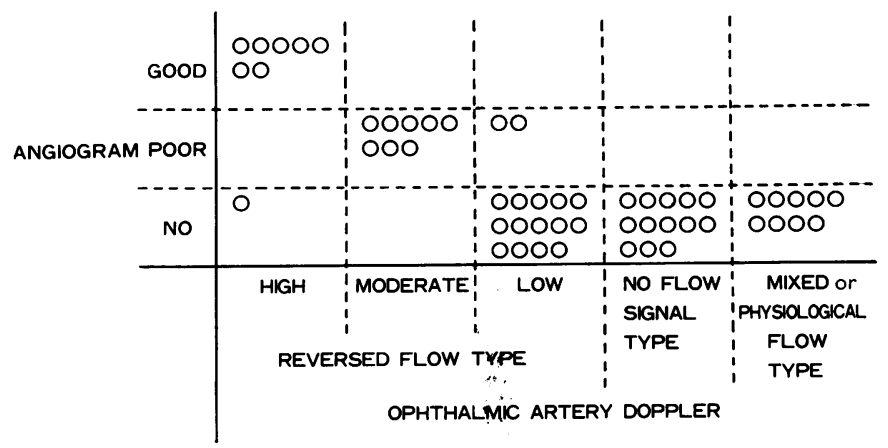

Fig. 8 Comparison of ultrasonic doppler with angiographic findings with regard to the ophthalmic collateral flow in 54 carotid artery occlusions.

Quantitative evaluation of blood flow by doppler ultrasound is instructive in the physiology and pathophysiology of blood flow. Some efforts have been made to determine blood flow volume by doppler ultrasound, but those seem to have been limited to experimental study or to biomedical engineering, and there have been few clinical studies (Doriot et al., 1975; Keller et al., 1976b; Fish, 1978).

From the results of the present study, it is clear that there are four types of ophthalmic blood flow in carotid artery occlusions-reversed, mixed, no flow, and physiological flow types. Moreover, the amount of reversed blood flow can be graded as high, moderate, or low. This semiquantitative evaluation by doppler ultrasound proved very reliable. The noncorrelation between the findings of angiogram and the doppler study in three patients were all the result of particular pathological events and never of inaccuracy of the ultrasonic technique.

We can demonstrate the clinical importance of the semiquantitative evaluation of ophthalmic collateral flow with three interesting examples. A 
reversed ophthalmic flow in a 72 year old man with internal carotid occlusion changed from low reversed flow to high reversed flow in the course of seven days of the acute stage according to ultrasonic doppler examination. His angiograms also changed from "no collateral flow" to "good collateral flow." In this patient, canalisation of the ophthalmic collateral flow was diagnosed by ultrasonic doppler study. A high reversed flow in the ophthalmic artery of a 52 year old man with internal carotid occlusion also changed to a low reversed flow during the course of 10 months according to doppler study. His angiograms also changed from "good collateral flow" to "no collateral flow." This means that ultrasonic doppler examination can diagnose an occlusion of the ophthalmic collateral flow. In the third case previously reported by Kaneda et al. (1978), recanalisation of the occluded carotid artery could be diagnosed noninvasively by the ultrasonic doppler technique.

The technique for detection of ophthalmic artery blood flow was superior to that for medial frontal artery blood flow for the purpose of quantitative evaluation of the ophthalmic collateral flow as well as for a decision regarding the direction of flow in the ophthalmic arterial territory (Kaneda et al., 1978). When ophthalmic artery blood flow was measured as in the present study, the findings of the angiograms and the doppler study disagreed in only three of 54 subjects. But when the blood flow of the medial frontal artery was measured and compared with the angiograms, there were as many as eight patients with no correlation. The reason may be that the diameter of the medial frontal artery varies more than that of the ophthalmic artery.

From a theoretical standpoint, the value of the maximum blood flow velocity at systole (S) is a very rough index of blood flow because it neglects the diameter of the ophthalmic artery, the angle between the artery and the ultrasonic beam, and the mean blood flow velocity. For more precise determination of the volume of blood flow, some theoretical considerations are necessary.

The absolute quantity of the blood flow volume in an artery (Q) is generally calculated by the following equation:

$$
\mathrm{Q}=\mathrm{C} \frac{\pi \mathrm{r}^{2}}{\cos \theta} \int_{0}^{t} \overline{\mathrm{V}}(\mathrm{t}) \mathrm{dt}
$$

where $\bar{V}(t)$ is the mean blood flow velocity in the line of the sound beam, $r$ is the radius of the artery, $\theta$ is the angle between the axis of the artery and the sound beam, and $C$ is a constant.

$\mathrm{V}(\mathrm{t}), \mathrm{r}$ and $\theta$ are the important data necessary for calculation of the absolute quantity of blood flow volume $(\mathrm{Q})$ : $\mathrm{r}$ and $\theta$ can be measured with the use of an ultrasonic duplex echo-doppler scanner (Barber et al., 1974) (Fig. 9). Mean blood
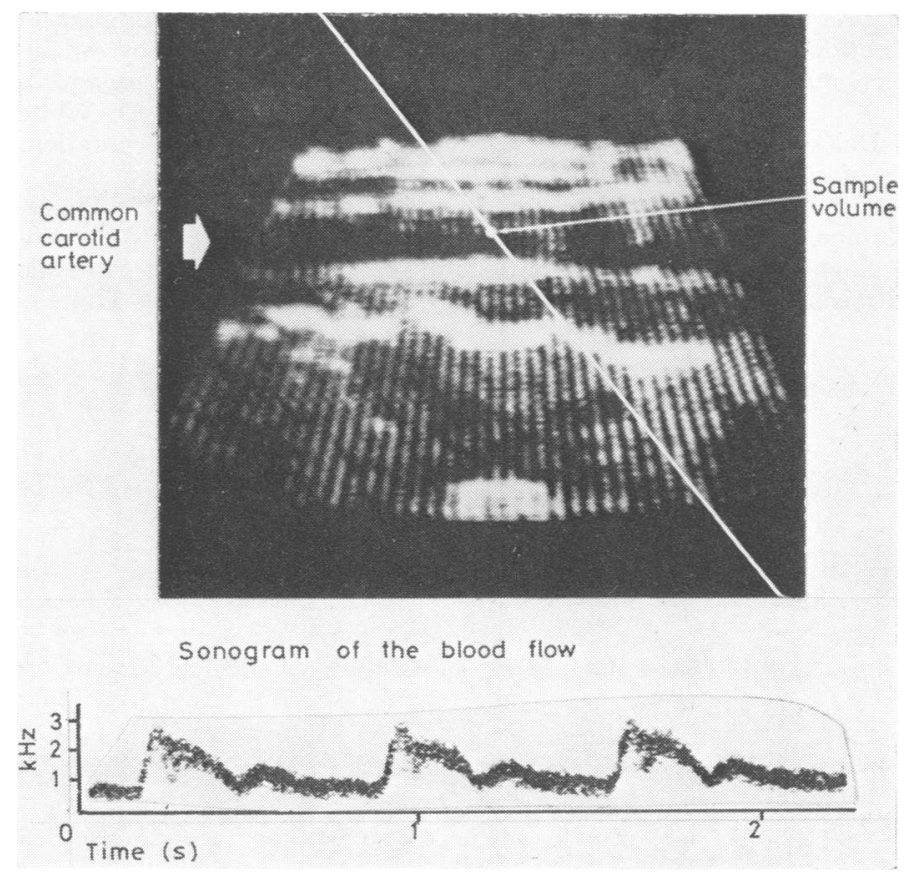

Fig. 9 Ultrasonic duplex echo-doppler scan showing a longitudinal sectional image of common carotid artery. The path of the sound beam is indicated by the oblique line (upper). Sound signal of the blood flow in the sample volume is detected and analysed by the sound spectrograph, giving a sonogram (lower). 
flow velocity in the line of the sound beam $(\mathrm{V}(\mathrm{t}))$ or, at least an approximate value of it, may be calculated from the data obtained from the sonogram of the blood flow. We hope, therefore, that the absolute quantity of the blood flow volume in an artery will be measured noninvasively in the near future by such an ultrasonic doppler instrument.

\section{References}

Barber, F. E., Baker, D. W., Nation, A. W. C. Strandness, D. E., and Reid, J. M. (1974). Ultrasonic duplex echo-Doppler Scanner. IEEE Transactions on Biomedical Engineering, 21, 109-113.

Doriot, P. A., Casty, M., Milakara, B., Anliker, M., Bollinger, A., and Siegenthaler, W. (1975). Quantitative analysis of flow conditions in simulated vessels and large human arteries and veins by means of ultrasound. In Ultrasonics in Medicine, pp. 160168. Edited by M. deVlieger, D. N. White, and V. R. McCready. American Elsevier: New York.

Fish, P. J. (1978). An integrated system for vessel imaging and blood flow measurement (abstract). Third European Congress on Ultrasonics in Medicine, pp. 179-180. Bologna.

Hyman, B. N. (1974). Doppler sonography. American Journal of Ophthalmology, 77, 227-231.

Kaneda, H., Irino, T., Minami, T., and Taneda, M. (1977). Diagnostic reliability of the percutaneous ultrasonic Doppler technique for vertebral arterial occlusive diseases. Stroke, 8, 571-579.

Kaneda, H., Irino, T., Arita, N., Minami, T., Taneda, M., and Shiraishi, J. (1978). Relationship between ophthalmic artery blood flow and recanalisation of occluded carotid artery-ultrasonic Doppler study. Stroke, 9, 360-363.

Katz, D. M., Smith, R. A., Otis, S. A., and Dalessio, D. J. (1976). Doppler sonography diagnosis of cerebrovascular disease. Stroke, 7, 439-444.

Keller, H., Meier, W., Yonekawa, Y., and Kumpe, D. (1976a). Non-invasive angiography for the diagnosis of carotid artery disease using Doppler ultrasound.
Stroke, 7, 354-363.

Keller, H. M., Meier, W. E., Anliker, M., and Kumpe, D. A. (1976b). Noninvasive measurement of velocity profiles and blood flow in the common carotid artery by pulsed Doppler ultrasound. Stroke, 7, 370-377.

Lye, C. R., Summer, D. S., and Strandness, D. E. (1976). The accuracy of the supraorbital Doppler examination in the diagnosis of hemodynamically significant carotid occlusive disease. Surgery, 79 , 42-45.

Maroon, J. C., Campbell, R. L., and Dyken, M. L. (1970). Internal carotid artery occlusion diagnosed by Doppler ultrasound. Stroke, 1, 122-127.

Müller, H. R. (1972). The diagnosis of internal carotid artery occlusion by directional Doppler sonography of the ophthalmic artery. Neurology (Minneapolis), 22, 816-823.

Müller, H. R. (1973). Directional Doppler sonography -a new technique to demonstrate flow reversal in the ophthalmic artery. Neuroradiology, 5, 91-94.

Satomura, S., and Kaneko, Z. (1960). Ultrasonic blood rheograph. Proceedings of the Third International Conference on Medical Electronics, pp. 254-258. London.

Shoumaker, R. D., and Bloch, S. (1978). Cerebrovascular evaluation: assessment of Doppler scanning of carotid arteries, ophthalmic Doppler flow and cervical bruits. Stroke, 9, 563-566.

von Reutern, G. M.. Budingen, H. J., Hennerici, M., Freund, H. J. (1976). Diagnose und Differenzierung von Stenosen und Verschlussen der Arteria carotis mit der Doppler-Sonographie. Archiv Psych:atrie Nervenkrankheit, 222, 191-207.

White, D. N., and Curry, G. R. (1978). A comparisonce of 424 carotid bifurcations examined by angio-? graphy and the Doppler echoflow. In Ultrasound in Medicine, vol. 4, pp. 363-376. Edited by D. White and E. A. Lyons. Plenum Publishing Corporation: New York.

Wise, G., Parker, J., and Burkholder, J. (1979). Supraorbital Doppler studies, carotid bruits, and arteriography in unilateral ocular or cerebral ischemic disorders. Neurology (Minneapolis), 29, 34-37. 